\title{
Pagando para vencer, parte 2: serialização, power creep e capitalismo tardio em Hearthstone
}

\section{Paying to win, part 2: serialization, power creep, and late capitalism in Hearthstone}

Thiago Falcãol

Daniel Marques ${ }^{2}$

Resumo: Este artigo discute o fenômeno de power creep no card game digital Hearthstone (Blizzard, 2012), problematizando-o a partir das perspectivas da midiatização, serialização e capitalismo tardio. O objetivo central é politizar a questão, apontando para os imbricamentos da indústria cultural contemporânea com as perspectivas epistemológicas e ontológicas do jogo enquanto chave interpretativa. Nesse sentido, empreendemos o esforço de revisar algumas teorias da midiatização, observando em particular o conceito nos videogames a partir da serialização. Partimos para uma análise empírica exploratória de relatos de jogadores profissionais no YouTube, revelando discursos que nos ajudam a localizar a agência do capitalismo tardio a partir da serialização e da midiatização. Por fim, reforçamos a necessidade de contemplar os pontos desvelados pela pesquisa na compreensão da indústria contemporânea do entretenimento, bem como a necessidade de (re)pensar os entendimentos particulares sobre jogos digitais e suas culturas.

Palavras-chave: card games; eSports; jogo; midiatização; capitalismo tardio.

Abstract: This paper discusses power creep in the digital game card Hearthstone (Blizzard, 2012), questioning it from the perspectives of mediatization, serialization and late capitalism. The goal is to politicize the issue by pointing

1 Universidade Federal da Paraíba (UFPB). João Pessoa, PB, Brasil. https://orcid.org/0000-0001-6302-2264 E-mail: thfalcao@cchla.ufpb.br

2 Universidade Federal do Recôncavo da Bahia (UFRB). Santo Amaro, BA, Brasil. https://orcid.org/0000-0002-7731-8581 E-mail: danielmarquescontato@gmail.com 
to the overlapping of contemporary cultural industry with game's epistemological and ontological perspectives. We undertake the effort to review some theories of mediatization, observing the particularities of serialization in games. To achieve the main goal, we set out for an exploratory empirical analysis of professional players discourse on YouTube, revealing traces that help us locate the agency of late capitalism and serialization in Hearthstone's power creep. Finally, we reinforce the need to scrutinize the contemporary entertainment industry, as well as the need to (re) think understandings about digital games and their cultures.

Keywords: card games; e-sports; game; mediatization; late capitalism. 


\section{Introdução}

O jogo, no aspecto mais amplo do termo, é um dos elementos centrais do desenvolvimento da cultura. O impulso criativo, a brincadeira e a apropriação são poderosos catalisadores agenciais, tanto na ação situada quanto para condições estruturais mais amplas. Esse jogo sem bordas, livre, sem regras; de um impulso que se (nos) aproxima da loucura, é imprescindível em qualquer análise mais completa de um espectro social qualquer. Esta discussão pressupõe que a compreensão das organizações sociais da contemporaneidade obriga uma observação através de condições epistemológicas que privilegiem o jogo como chave de leitura.

Esta retórica sugere um desvio pela ideia de jogo, que ao se apresentar como mediador para uma compreensão sociológica modifica-a. Intenta-se, portanto, resgatar a noção de jogo do status de irrelevância a ele conferido pelo raciocínio positivista e pela Modernidade, contextos que ainda ecoam em suas representações, e posicioná-la ao lado dos pilares epistemológicos contemporâneos; das chaves de leitura que nos utilizamos para compreender a sociedade dos dias de hoje. Trata-se de uma discussão ampla, um esforço de longo prazo sobre afetações na tessitura do social que convocam um escrutínio das práticas agenciais e materiais envolvidas neste processo.

Assim, ao mesmo tempo em que busca empreender um esforço de compreensão de dinâmicas microagenciais ao discutir o fenômeno conhecido como power creep e a forma como este acontece no card game Hearthstone ${ }^{3}$ (BLIZZARD ENTERTAINMENT, 2012), este artigo procura sublinhar o papel das dinâmicas comuns ao capitalismo tardio (JAMESON, 1991) e como estas são particularmente relevantes no estudo de uma cultura contemporânea dos games.

O power creep, um fenômeno que acontece em jogos publicados de forma serial, se assemelha à ideia de obsolescência programada e acontece quando certos recursos dentro destes sistemas se tornam obsoletos e demandam uma substituição que só pode ser executada através de relações de consumo. A questão ilustrada por este fenômeno diz respeito à serialização da experiência dos meios de comunicação 
de forma mais ampla, e remete a dois processos que são largamente discutidos no âmbito da sociologia da mídia: (1) a produção de bens simbólicos em escala industrial, discutida a partir da noção de indústria cultural e das derivações do conceito e de seus problemas; e (2) o fenômeno da midiatização, através do qual instituições e práticas sociais vêm sendo modificadas pela ubiquidade comunicacional.

Este artigo oferece continuidade a uma discussão travada anteriormente (FALCÃO; MARQUES, 2017), que conecta dois aspectos da experiência contemporânea do jogo: um de ordem social, mais amplo e genérico, que cria ecos e referências culturais; e outro de ordem técnica, comprometida com o aspecto interno à mídia, sua materialidade. Nossa proposta é demonstrar como o fenômeno do power creep emerge enquanto consequência direta do processo de midiatização experimentado pelos jogos atuais, e reafirmar a intenção de problematizar a relação entre a indústria dos jogos e dinâmicas mercadológicas que implicam relações de poder. Para tanto, torna-se necessário observar uma cadeia de práticas que vão desde o game design até as políticas institucionais da Blizzard, passando pela conformação dos ambientes discursivos mobilizados pelo jogo. Observamos essas práticas a partir de uma análise exploratória de vídeos no YouTube sobre o fenômeno de power creep: relatos de jogadores profissionais, comentadores e funcionários da Blizzard discutindo o tema e suas implicações. Em vias de conclusão, apontamos a conexão dos fenômenos de obsolescência inerentes a Hearthstone a estruturas de agenciamento globais, à cultura do jogo e ao mercado de entretenimento contemporâneo.

\section{Midiatização e lógica das mídias}

O conceito de midiatização vem sendo amplamente trabalhado no campo da comunicação nas últimas décadas, produzindo entendimentos polissêmicos sobre seus pressupostos e aplicações. Os estudos da midiatização surgem a partir do reconhecimento do crescente imbricamento entre as instituições e campos sociais - religião, política, educação etc. - com a mídia, sua lógica e práticas (COULDRY, 2004). As diferentes 
abordagens à teoria da midiatização produzem afetações particulares do conceito. Verón (2014), por exemplo, parte do entendimento da midiatização sob um viés semioantropológico, sendo "resultado operacional de uma dimensão nuclear de nossa espécie biológica, mais precisamente, sua capacidade de semiose" (VERÓN, 2014, p. 14). Essa operacionalização resulta na constituição de fenômenos midiáticos, que seriam a exteriorização dos processos mentais na forma de dispositivos materiais. A midiatização consistiria, para Verón, na estabilização desses fenômenos midiáticos à medida que sua configuração, uso e prática se institucionaliza. Cria-se, portanto, "um lugar e tempo particular ao redor de um dispositivo de comunicação” (VERÓN, 2014, p. 16). É importante, portanto, considerar a implicação das teorias da midiatização na análise dos jogos digitais na contemporaneidade. A experiência do “jogar” e, consequentemente, a cadeia produtiva - do design ao consumo via streaming - é afetada e tensionada pelo ambiente midiatizado do jogo. Para um jogo digital obter sucesso comercial, portanto, é preciso apropriar-se do ambiente midiatizado onde circulam, hoje, os objetos da cultura. Hearthstone, por exemplo, figura atualmente como o décimo quarto jogo mais popular no Twitch ${ }^{4}$, junto a outros jogos da Blizzard. Overwatch e World of Warcraft, outros jogos da mesma empresa, figuram no sétimo e oitavo lugar, respectivamente. Esses dados não são de forma alguma aleatórios, e apontam para uma intencionalidade - by design por parte da Blizzard em midiatizar suas experiências de jogo.

A busca pela midiatização de corporações como a Blizzard aponta para os processos de estabilização tratados por Verón, sugerindo uma operacionalização particular que se coalesce na ideia de institucionalidade. Sob esse viés entende-se que a mídia passa a figurar enquanto instituição social cujos processos não são somente pautados pelos campos sociais estabelecidos, mas passa a possuir legitimidade e autonomia próprias. Passa, assim, a afetar e tensionar outros pontos do tecido social. Para Braga (2012), o imbricamento dos meios de comunicação

4 Trata-se da plataforma de streaming mais popular e amplamente adotada por streamers e jogadores profissionais. Dados e métricas podem ser encontradas aqui: https:/www. twitchmetrics.net 
com os campos sociais pressupõe refletir também acerca dos conceitos de circulação, mediação, recepção, produção e ambiência midiática. A pesquisa em midiatização, portanto, funcionaria como uma espécie de guarda-chuva, capaz de abarcar as diferentes lógicas, mediações, circuitos e dispositivos comunicativos oriundos da institucionalização da mídia e a produção de novos ambientes midiáticos. Essa hipótese parte do princípio de que habitamos um mundo midiatizado (BASTOS, 2012, p. 68), marcado pela influência da mídia na constituição da cultura e sociedade. A “midiatização" do mundo, portanto, pressupõe não só o reconhecimento da lógica da mídia nos artefatos e instituições sociais, mas também a vinculação material dos dispositivos midiáticos na estrutura dos fenômenos contemporâneos.

Num mundo midiatizado não seríamos mais capazes de analisar os campos sociais, seus processos e produtos descolados da ação das mídias: trata-se de uma hibridização completa e indelével. Na indústria dos jogos digitais, de maneira geral, é possível verificar a presença dessa hipótese. Cada vez mais o projeto dos jogos - seu game design, portanto - busca a integração de processos de midiatização. Esse imbricamento é profundo e observável materialmente, inclusive, em consoles contemporâneos. Controles de consoles como PlayStation 4 e Nintendo Switch possuem botões dedicados para compartilhamento de conteúdo (Figura 1), e os sistemas operacionais desses consoles tendem a buscar integração com plataformas como YouTube, Facebook e Twitch. As mudanças materiais, portanto, na estrutura física dos consoles e no funcionamento de seus sistemas operacionais e interface aponta para a naturalização dos fenômenos de midiatização quando consideramos sua presença no game design. 
Figura 1 - Botões de compartilhamento de conteúdo em consoles
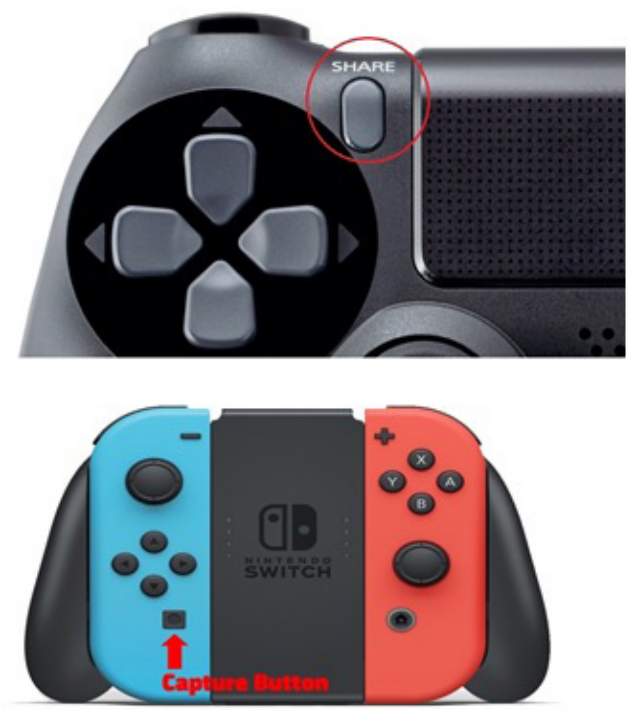

Fonte: https://boards.fireden.net/v/thread/369558869/

Há sempre uma negociação entre o que os autores denominam de lógica da mídia para as outras lógicas institucionais da cultura e sociedade. Não se trata de admitir uma sobreposição ou colonização das lógicas institucionais pela mídia, mas compreender que a formação de novos ambientes midiáticos produz afetação tanto na mídia quanto nos demais campos. Um exemplo interessante é documentado por Turtiainen et al. (2018), que evidencia a "esportificação" nos eSports, como defendido por Falcão e Marques (2017), de forma a aproximá-los das lógicas dos esportes tradicionais. Os autores (TURTIAINEN et al., 2018) empenham uma análise da cobertura da Overwatch World Cup 2016, comparando-a com a Copa do Mundo FIFA 2014, expondo semelhanças e diferenças na forma como os dois fenômenos abordam práticas relacionadas à mídia.

Por um lado, o modus operandi das competições esportivas - esportificação - aparece no esforço da Blizzard Entertainment em aproximar o formato da Overwatch World Cup ao de torneios e campeonatos esportivos tradicionalmente reconhecidos como tal, de forma a facilitar as 
práticas de recepção e circulação das audiências envolvidas (BRAGA, 2012). Isso se verifica materialmente na estrutura do campeonato, na formatação dos times, uniformes, divisão por países, fases, métodos de classificação etc. Também se faz sentir a lógica da mídia - midiatização -, na medida em que há um amplo esforço em aproximar a transmissão da Overwatch World Cup de outros produtos midiáticos do campo do esporte. O formato do estúdio, a enunciação dos apresentadores e comentaristas, as interfaces gráfico-visuais de apresentação de jogadas e placares etc. Esse próprio formato de broadcast já é resultado da hibridização das lógicas da mídia com as lógicas do esporte.

O caminho através das teorias da midiatização nos ajuda a compreender que não se trata de um fenômeno tecnodeterminista: entender a midiatização pressupõe observar suas institucionalidades (HJARVARD, 2014), seu caráter semiótico (VERÓN, 2014), as práticas sociais cotidianas (HEPP, 2013) e a hibridização entre sociedade e técnica (COULDRY; HEPP, 2016). Essa visada polissêmica nos ajuda a compreender os fenômenos sociais - e da comunicação - de forma contextualizada, observando a configuração dos ambientes midiáticos e mundos midiatizados em sua materialidade.

\section{Midiatização e a experiência do jogo e da brincadeira}

Stig Hjarvard devota, em The mediatization of culture and society (2013), um capítulo à discussão sobre como a brincadeira 5 foi midiatizada, tanto direta como indiretamente, o que implica na noção de que o ato de brincar foi transposto para um horizonte no qual não há condições de empreendê-lo de forma alheia aos meios de comunicação. Nos interessa empreender uma crítica à forma como o autor sugere que o videogame é experimentado em caráter hegemônico, a ponto de seu consumo ser equiparado à prática da brincadeira per se. Nos debruçamos sobre

5 Play, no original, que é uma palavra de difícil tradução e que possui mais sentidos do que a língua portuguesa consegue condensar. As traduções aqui variam entre jogo e brincadeira, atentando para contextualidades específicas. Ver Juul (2005) e Falcão (2010), no que diz respeito ao "problema da linguagem". 
a argumentação de Hjarvard a partir de dois posicionamentos que são certamente imperativos à redução empreendida à atividade lúdica.

O primeiro deles diz respeito a uma dimensão da representação, e está relacionado à forma como, na contemporaneidade, os produtos infanto-juvenis se encontram sempre relacionados à esfera dos meios de comunicação de massa. Pode-se observar este fenômeno, inclusive, em produtos costumeiramente endereçados sob a alcunha de geek, desenhados para um público que Hjarvard considera adulto, mas que permanece jovem por mais tempo - ASYL $^{6}$ - e sublinha, por exemplo, que "os brinquedos de hoje dificilmente lembram os objetos do mundo real" (2013, p. 167) e "fazem parte de um mundo de fantasia" (p. 168), em um movimento que ignora de forma relevante tanto a constituição histórica das práticas do jogo, em seu aspecto mais amplo, quanto discussões recentes acerca da materialidade deste. É necessário considerar que a relação entre brincadeira e atributos ficcionais não é particularmente nova ou, sequer, um produto do processo de midiatização: subsistem no faz de conta (mimicry) representações e diálogos com as franquias audiovisuais contemporâneas, mas não se pode esquecer que sempre fomos, em nossas brincadeiras, piratas, astronautas, cavaleiros.

A segunda posição diz respeito a uma leitura que identifica, no raciocínio de Hjarvard (2013), aquilo que Bruno Latour (2012) classifica como pensamento purificador. A despeito do dado argumento se expressar de forma factual, como uma leitura de um contexto social específico, é sensível uma clara urgência em se categorizar a relação entre trabalho e lazer no argumento do autor. Não à toa, por exemplo, este discute sua análise do processo de midiatização da brincadeira a partir de uma ideia de expectativa associada às faixas etárias específicas elencadas. Estar atento a essas posições é fundamental para que se possa repensar o lugar do jogo enquanto objeto da comunicação. A questão aqui diz menos respeito à formação de contornos para um campo que se debruce sobre os games, e mais à necessidade de se reconhecer que um dos grandes feitos do capitalismo tardio (JAMESON, 1991) foi

6 Sigla para Adults staying Young longer. 
rearranjar as fronteiras e as percepções com relação ao que percebemos, hoje, como as dicotomias da modernidade. Pensar desta forma implica reconhecer que a celeridade e imediatez com a qual experimentamos a contemporaneidade é uma consequência direta da forma como as dinâmicas do capital se alastraram por todas as esferas da vida social.

Assim, a urgência em perceber de que forma o jogo se torna um objeto imperativo para as epistemologias comprometidas com a interpretação do social jaz no simples fato de que este domínio, antes livre, no sentido em que não é obrigatório; separado, à medida que é circunscrito a um tempo e espaço limitados; incerto, pois seu curso não pode ser determinado e improdutivo, porque não pode criar bens ou riqueza, hoje se apresenta como mais um dos muitos contextos colonizados pelo raciocínio capitalista. O argumento de Hjarvard (2013), ao executar a mesma purificação encontrada em autores do início do século XX, sublinha o aspecto mercadológico imbuído na experiência do jogo ao simplificar sua relação e a forma como este é um poderoso vetor agencial na sociedade contemporânea.

Hearthstone, por exemplo, ao mesmo tempo em que faz uso de personagens e histórias tecidas ao longo das décadas pelos vários produtos da franquia de Warcraft (Blizzard Entertainment, 1994-2018), é projetado como um produto de uso contínuo e frequentemente atualizável. Este tipo de mecânica busca transformar a experiência do jogo não em um momento de consumo, mas em uma experiência longitudinal na qual um jogador está, de certa forma, sempre em diálogo com o produto. A Blizzard possui uma série de estratégias que dão vazão a este comportamento, fomentando a produção e circulação do jogo a partir de uma relação criada com a comunidade. Esse comportamento é prescrito pela empresa, ou seja, integra seu programa de ação (LATOUR, 2012). Ademais, utiliza-se das materialidades produzidas pelo espraiamento do fenômeno da midiatização, conforme discutido previamente. A relação que nos interessa particularmente neste artigo, contudo, é uma de ordem mais direta: uma estabelecida entre o jogador e o jogo de forma mais sistemática, não contemplando as possibilidades paratextuais, 
narrativas incutidas no produto, mas que é parte, também, deste movimento de midiatização comercial.

Retomamos nossa hipótese, portanto, de que o power creep experimentado em um jogo como Hearthstone é uma consequência direta da serialização imposta pelas dinâmicas do capitalismo tardio, e que a forma mais adequada de compreender este fenômeno e suas repercussões é perceber de que forma se empreende, nesta relação entre jogo e jogador, uma dinâmica facilmente reconhecida como de midiatização.

\section{Interlúdio: sobre serialização}

Hearthstone, como discutido em Falcão e Marques (2017), é um jogo de cartas publicado e atualizado continuamente pela Blizzard Entertainment desde 2012, e que oferece ao jogador uma coleção de centenas de cartas que precisam ser adquiridas a partir de formas regidas por sólidas relações econômicas entre tempo de jogo e dinâmicas de pay to play, para, em seguida, organizar 30 delas em um baralho (deck) e, então, estar apto a competir com outros jogadores. É um jogo competitivo (agôn), cujo objetivo é a vitória através de uma série de movimentos estratégicos, mas que possui diversos elementos de aleatoriedade (alea) em sua jogabilidade, que balizam as decisões, estratégias e filosofias do jogo em si.

Cada expansão de Hearthstone transforma o jogo apenas um pouco. O suficiente para que a mudança seja sentida, mas não para que se possa alegar que o jogo é outro. Essa lógica no game design dá vazão a uma ligação muito forte entre Hearthstone e outros domínios da indústria cultural. O jogo não apenas dialoga com as franquias da Blizzard, mas se conecta a todo um espectro cultural, estabelecendo um rico diálogo com a cultura dos jogos, com a cultura de massa e com sua própria história. Ao se apropriar do mundo ficcional de Warcraft, discutido por Falcão (2014) e Aarseth (2009), entre outros, Hearthstone ganha um rico universo que, aos poucos, vai sendo trabalhado através de várias nuances que (1) paratextualizam a experiência do jogo e, finalmente, (2) oferecem um suporte narrativo para o que poderia ser apenas um jogo de cartas qualquer. 
Por estar subscrito a uma dinâmica de serialização, Hearthstone publica, a cada três meses, novas cartas que, por vezes, tornam outras cartas obsoletas no que tange a participação competitiva do jogador no contexto do jogo. É importante considerar que a dimensão competitiva é fortemente prescrita pelo fenômeno de midiatização de Hearthstone, os streamers, youtubers e outros digital influencers do jogo são, comumente, pro players. Essa dimensão seria interessante por si só se discutida apenas a partir do design; e quando se adiciona a camada de problematização política - aqui discutida a partir de como o jogo é consumido, ela se torna uma questão legítima e imperativa para a compreensão não apenas da relação entre jogo e jogador, mas de uma nuance social que envolve legítimas relações de poder que são movimentadas a partir de decisões que afetam o estado de jogo e, assim, agenciam comportamentos ao seu redor. A partir de agora, discutiremos esta dimensão de forma mais sistematizada.

\section{Procedimentos metodológicos}

Este trabalho, assim, para além de estabelecer uma discussão de ordem epistemológica acerca das práticas atuais do jogo contemporâneo, se alicerça sobre um viés teórico-metodológico exploratório. Nossa observação foi realizada a partir de uma análise descritiva do conteúdo que toma como recorte vídeos publicados no YouTube que possuem os termos "power creep" e "Hearthstone" como chaves de indexação. Embora a busca inicial tenha revelado uma quantidade maior de vídeos, optamos por analisar somente aqueles com, no mínimo, 40.000 visualizações e gravados em inglês, o que oferece, em nossa opinião, maior consistência à amostra, uma vez que esta concentra um conteúdo de maior relevância para os usuários. É possível visualizar a lista completa dos vídeos analisados no Quadro 1. 
Quadro 1 - Vídeos analisados

\begin{tabular}{|c|c|c|c|c|c|}
\hline TÍTULO & VIEWS & USER & PERFIL & TEMPO & DATA \\
\hline $\begin{array}{l}\text { Power Creep in } \\
\text { Hearthstone - What } \\
\text { It Teaches Us About } \\
\text { Games - Extra Credits }\end{array}$ & 643.448 & $\begin{array}{l}\text { Extra } \\
\text { Credits }\end{array}$ & $\begin{array}{l}\text { Canal informativo } \\
\text { com conteúdo sobre } \\
\text { games, história, } \\
\text { ficção científica e } \\
\text { mitologia. }\end{array}$ & 07:08 & Set/15 \\
\hline $\begin{array}{l}{[\text { Hearthstone] Ben }} \\
\text { Brode \& Kripp On } \\
\text { Bad Cards }\end{array}$ & 624.337 & Kripparrian & $\begin{array}{l}\text { Streamer e comen- } \\
\text { tador de HS. É, } \\
\text { atualmente, um dos } \\
\text { mais populares em } \\
\text { HS no Twitch. }\end{array}$ & $30: 46$ & Jun/15 \\
\hline
\end{tabular}

\begin{tabular}{|c|c|c|c|c|c|}
\hline $\begin{array}{l}\text { [Hearthstone] How I'd } \\
\text { Improve HS }\end{array}$ & 319.814 & Kripparrian & $\begin{array}{l}\text { Steamer e } \\
\text { comentador de HS. } \\
\text { É, atualmente, um } \\
\text { dos mais populares } \\
\text { em HS no Twitch. }\end{array}$ & $21: 12$ & Dez/15 \\
\hline $\begin{array}{l}\text { [Hearthstone] Power } \\
\text { Creep \& Buffing Bad } \\
\text { Cards }\end{array}$ & 255.012 & Kripparrian & $\begin{array}{l}\text { Steamer e } \\
\text { comentador de HS. } \\
\text { É, atualmente, um } \\
\text { dos mais populares } \\
\text { em HS no Twitch. }\end{array}$ & $14: 22$ & Ago/15 \\
\hline $\begin{array}{l}\text { [Hearthstone] Doing } \\
\text { Your Best With Trash }\end{array}$ & 230.379 & Kripparrian & $\begin{array}{l}\text { Steamer e } \\
\text { comentador de HS. } \\
\text { É, atualmente, um } \\
\text { dos mais populares } \\
\text { em HS no Twitch. }\end{array}$ & $17: 27$ & Jan/17 \\
\hline $\begin{array}{l}\text { Designer Insights with } \\
\text { Ben Brode: The Dark } \\
\text { Side of Releasing New } \\
\text { Content }\end{array}$ & 195.986 & Hearthstone & $\begin{array}{l}\text { Perfil oficial da } \\
\text { Blizzard destinado a } \\
\text { conteúdo sobre HS. }\end{array}$ & $6: 53$ & Set/15 \\
\hline $\begin{array}{l}\text { Hearthstone: Trump } \\
\text { Talks - Card Design, } \\
\text { Power Creep \& The } \\
\text { Grand Tournament }\end{array}$ & 159.275 & Trump & $\begin{array}{l}\text { Jogador profissional } \\
\text { e streamer de HS. }\end{array}$ & 13:03 & Ago/15 \\
\hline $\begin{array}{l}\text { POWER CREEPIN' } \\
\text { MY HEARTHSTONE }\end{array}$ & 76.564 & Noxious & $\begin{array}{l}\text { Ex-jogador } \\
\text { profissional de HS. } \\
\text { Atualmente dedica- } \\
\text { se a MTG Arena. }\end{array}$ & $17: 30$ & Ago/15 \\
\hline $\begin{array}{l}\text { Thoughts on Power } \\
\text { Creep in Hearthstone }\end{array}$ & 41.064 & Noxious & $\begin{array}{l}\text { Ex-jogador } \\
\text { profissional de HS. } \\
\text { Atualmente dedica- } \\
\text { se a MTG Arena. }\end{array}$ & 26:08 & Nov/14 \\
\hline
\end{tabular}

Fonte: Autoria própria 
A análise não partiu de categorias já estabelecidas. Pelo contrário, nosso objetivo analítico reside na observação de quais posições os produtores desses vídeos - majoritariamente jogadores profissionais e analistas de Hearthstone - assumem em relação ao fenômeno de power creep. Em outras palavras, quais sentidos gerais estes sujeitos atribuem ao fenômeno? Como essa produção de sentido nos ajuda a problematizar aspectos da política de produção, circulação e negociação de bens virtuais em Hearthstone? E, para além disso, como esses discursos nos ajudam a localizar a agência do capitalismo tardio a partir da serialização e da midiatização do jogo.

Acreditamos que a análise de conteúdo oferece uma compreensão importante sobre como o fenômeno de power creep é percebido pela comunidade de usuários interessada, o que nos ajuda a problematizar o seu papel nas políticas de conteúdo e estratégias político-econômicas da Blizzard. Nesse sentido, acreditamos que esse trabalho expande e complementa o esforço de pesquisa realizado anteriormente (FALCÃO; MARQUES, 2017), pois elucida outra dimensão da circulação de bens virtuais em Hearthstone. Assim como no trabalho anterior, entendemos Hearthstone e os bens virtuais oriundos desse dispositivo enquanto objetos-rede (LATOUR, 2012), cuja agência e mediação se faz sentir para além da sua materialidade imediata, embora indissociável desta.

\section{Power creep em Hearthstone}

O fenômeno de power creep não é exclusivo a Hearthstone. Trata-se de um processo que tende a afetar qualquer jogo digital que passe por ciclos de atualização e expansão. O processo, contudo, se torna mais crítico em jogos que possuem foco na competição multiplayer, tendo em vista que a diferença de poder só passa a ser sentida em relação ao desempenho de outros jogadores. No caso de Hearthstone, é possível verificar a ocorrência de power creep quando o lançamento de novas cartas faz com que jogadores abandonem decks e cartas antigas com o intuito de permanecerem competitivos. Um exemplo claro de power creep pode ser visualizado na Figura 2. 
Figura 2 - Exemplo de power creep em Hearthstone

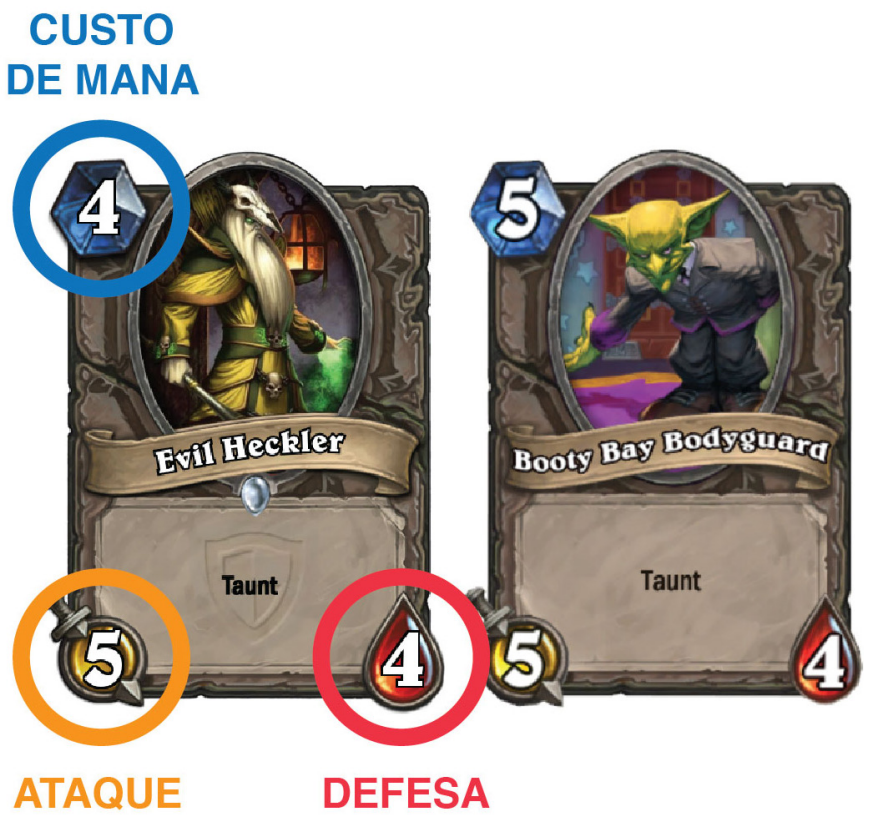

Fonte: Adaptado de Blizzard Entretainment

As duas cartas possuem os mesmos atributos básicos: cinco pontos de ataque e quatro pontos de vida, além da habilidade taunt - provocar. Entretanto, é possível perceber que a primeira - Evil Heckler (EH) - requer um cristal de mana a menos do que Booty Bay Bodyguard (BBB) para ser jogada. Essa diferença faz com que EH seja objetivamente melhor que BBB em qualquer cenário possível de jogo, já que ele precisa de menos recursos para ser ativado. Caso o jogador possua as duas cartas e deseje encaixar alguma em seu deck, EH se torna a escolha óbvia. A diferença crucial está no seguinte aspecto: enquanto BBB compõe a coleção de cartas básicas do jogo, à qual os jogadores podem ter acesso sem a mobilização de recursos financeiros, EH integra uma expansão paga (The Grand Toumament, lançada em 2015). Seja com dinheiro 
ou com recursos monetários do jogo ${ }^{7}$, a obtenção de EH exigirá tempo e esforço dos jogadores. Embora nenhuma das cartas discutidas acima sejam utilizadas no cenário competitivo, o exemplo nos ajuda a entender o fenômeno e ilustrar algumas questões. A barreira monetária é uma delas, conforme aponta Noxious, ex-jogador profissional, analista e comentador de Hearthstone:

Um argumento que é surge com muita frequência quando vejo pessoas defenderem o power creep que afeta as cartas básicas (...) As pessoas dizem coisas como: "O Booty Bay Bodyguard é uma carta grátis, o Evil Heckler não é uma carta grátis, então tudo bem se ela for um um pouco melhor que a carta grátis porque você percebe que cartas gratuitas não são as melhores". Bem, isso não tem nada a ver com o equilíbrio do jogo! (NOXIOUS, 2014, Tradução do autor ${ }^{8}$ )

No lado de quem recebe o design, eles (os desenvolvedores) basicamente lançam cartas que tornam cartas antigas obsoletas. Eles lançam na minha cara, especialmente quando há uma barreira monetária entre BBB (Booty Bay Bodyguard) e o 5/4 com Provocar. (NOXIOUS, 2015, Tradução do autor $^{9}$ )

A relação pontuada por Noxious entre cartas gratuitas e pagas se estabelece, como já dito, através do sistema de expansões. Esse sistema corrobora para que o ambiente do jogo esteja em constante atualização, com novos decks e estratégias competitivas surgindo e desaparecendo. Aqui está a primeira pista para visualizar os fenômenos de midiatização e, consequentemente, serialização em Hearthstone. A barreira monetária descrita por Noxious é envolta em uma carapaça midiática produzida

7 O processo de obtenção, circulação e negociação dos bens virtuais em Hearthstone foi amplamente discutido em Falcão e Marques (2017).

8 Citação original: An argument that is brought up very frequently when I read people defend the power creep that happen over basic cards (...) People are like: "Booty Bay Bodyguard is a free card, Evil Heckler is not a free card so it's fine if it's a bit better than the free card because than you realize that free cards are not the best". Well that has nothing to do with the game balance itself!

9 Citação original: On the receiving end of the design, they basically throw cards that obsolete older cards. They have it thrown in my face, especially when there is a monetary barrier between $\mathrm{BBB}$ and the $5 / 4$ with taunt. 
pela Blizzard de forma a obscurecer os processos de monetização envolvidos na aquisição das novas cartas. Não se trata também, como aponta Noxious, de questões de balanceamento do jogo, mas sim da filosofia que rege a modulação do ambiente de Hearthstone.

A mecânica de expansões constitui um dispositivo, uma rede que mobiliza a Blizzard, a equipe de Hearthstone e a comunidade de jogadores em diferentes práticas. É realizado um esforço de comunicação e narrativização - nos termos de Hjarvard (2013) - para que as novas expansões sejam desejadas: lançamento de vídeos promocionais no YouTube, parcerias com jogadores populares para revelação de novas cartas, lançamentos de HQs e contos que contextualizam o universo narrativo da próxima expansão etc. Há um forte entrelaçamento do que se reconhece como lógicas da mídia e o sistema de expansões. Esse esforço é reconhecido por streamers como Kripparrian:

A Blizzard está realmente fazendo um trabalho muito bom na produção de conteúdo. Essa expansão tem 132 cartas e, bem, foi desenvolvida no tempo padrão das expansões (...) O que isso quer dizer, uma média de 3 ou 4 cartões por dia? Eles estão realmente se esforçando bastante. (KRIPPARRIAN, 2015a, Tradução do autor ${ }^{10}$ )

Kripparrian explora esse argumento ao propor uma saída para o problema de power creep: atualizar cartas antigas, aumentando seu poder (buff). Para ele, os jogadores estariam interessados majoritariamente na introdução de novas cartas que, por sua vez, produzam novos tipos de deck e estratégias de jogo: sejam cartas inéditas ou cartas antigas reformuladas e/ou melhoradas. Seguindo seu argumento, esse resultado seria mais fácil de alcançar a partir do incremento no poder de cartas antigas que tenham caído em desuso. Ao fazer isso, argumenta Kripparrian, a Blizzard impulsionaria a dinâmica do jogo, já que a realização de melhorias (buffs) ou decrementos (nerfs) no nível de poder

10 Citação original: Blizzard is actually doing a really good job pushing up content. This expansion has 132 cards and well, at regular expansion time (...) What is that like, an average of like 3 or 4 cards a day? They are popping up pretty big time. 
de determinadas cartas ajudaria a produzir esse ambiente "caótico" e "criativo" ao qual Kripparrian se refere.

Essa posição nos parece interessante, pois desloca a atenção dos jogadores de uma conexão narrativa com o universo do jogo - e consequentemente as estratégias de serialização do conteúdo ficcional - para a sensação de novidade, ao ter cartas reformuladas à disposição no momento de construção dos decks. O argumento a favor do buff em cartas com baixo nível de poder, portanto, desconsidera os aspectos narrativos mobilizados por novas expansões:

(...) Eu acho que a maioria dos jogadores que jogam Hearthstone a longo prazo realmente não se importam se a expansão introduz novas cartas como Ragnaros ou Nefarian, o que importa é que novas cartas sejam introduzidas (...) eu acho que seria muito fácil alcançar esse objetivo apenas alterando cartas ruins pré-existentes. (KRIPPARRIAN, 2015b, Tradução do autor ${ }^{11}$ )

Em resposta a esse argumento, Ben Brode - ex-diretor-geral de Hearthstone - problematiza a ideia de que melhorar uma carta antiga seria mais fácil e/ou simples do que produzir uma carta nova. $\mathrm{O}$ argumento de Ben Brode implica que se há algum novo feature de design interessante para o ecossistema de Hearthstone - mecânicas, habilidades, modos de jogo etc. -, a Blizzard prefere introduzir esse elemento enquanto conteúdo inédito. Novas cartas que integram novas expansões, portanto, fazem com que o ambiente e ecossistema do jogo cresçam constantemente. Em outras palavras, para Ben Brode seria mais interessante expandir a quantidade total de conteúdo disponível para os jogadores em detrimento ao processo de remanejo de conteúdo antigo.

Essa disputa entre a Blizzard e Kripparrian nos ajuda a entender algumas questões. Nos parece que tanto a Blizzard quanto a comunidade de jogadores possuem interesse na manutenção de Hearthstone

11 Citação original: (...) I think that most players who play Hearthstone in the long end don't really care if the expansion introduces new cards like Ragnaros or Nefarian, they only care if new cards are being introduced (...) I think that would be very easy to do by just changing bad cards. 
enquanto um ambiente flexível, criativo e orgânico, no qual estratégias de jogo estão sempre em desenvolvimento e se modificando ao longo do tempo. Entretanto, enquanto a Blizzard defende esse processo a partir do lançamento constante de novo conteúdo, Kripparrian e outros interlocutores (como Noxious e Trump) sugerem que alterações em cartas antigas produziriam efeitos semelhantes, além de acarretar menor desgaste econômico por parte dos jogadores.

É evidente que a implementação de buffs e nerfs não corrobora com a estratégia econômica da Blizzard: capitalizar a partir da serialização. A cada nova expansão lançada há um grande investimento na divulgação e comercialização de pacotes promocionais, a partir dos quais os jogadores podem ter acesso a conteúdos exclusivos, maior quantidade de cartas novas, itens cosméticos etc. Não obstante, percebem-se processos de naturalização do power creep pela Blizzard: pode-se observar o fenômeno como materializado na filosofia de game design da empresa. Pensar desta forma implica conceber que subsiste uma relação agencial de muita força, considerando o quão importante a Blizzard é enquanto ator nessa rede sociotécnica.

Nesse sentido, é evidente que a Blizzard obscurece seu interesse mercadológico, envolvendo-o em um discurso voltado para aspectos técnicos de game design e o bem-estar idealizado da comunidade de jogadores. Ora os argumentos de Ben Brode versam sobre aspectos materiais do objeto (balanceamento, conteúdo narrativo, novas mecânicas etc.), ora sobre a percepção dos jogadores e a sua satisfação com o estado das coisas em Hearthstone. Por outro lado, em nenhum momento Ben Brode menciona os impactos que novas cartas e expansões produzem no cenário do jogo, estabelecendo assim um sistema de demanda por cartas mais competitivas. Kripparrian explicita esse ponto:

Algumas coisas realmente impedem que novos jogadores joguem Hearthstone e uma dessas coisas é a quantidade de dinheiro e o comprometimento que você realmente precisa para atingir um nível aceitável e tornar-se capaz de jogar uma partida competitiva. Isso é algo que a Blizzard constantemente ignora a cada expansão. Com muito 
mais cartas a serem adquiridas, com cartas de difícil acesso em diferentes tipos de pacotes (...) Com cada expansão, cada jogador de Hearthstone terá mais dificuldade para recuperar o atraso, terá mais dificuldade de atingir um nível adequado de fair-play. É realmente péssimo para eles. (KRIPPARRIAN, 2015c, Tradução do autor ${ }^{12}$ )

Para além de pensar o power creep enquanto fenômeno relacionado à dinâmica de balanceamento do jogo, precisamos politizá-lo, problematizando-o a partir das estratégias econômicas dos desenvolvedores. Esse ponto se ausenta no discurso de Ben Brode, o designer define power creep a partir de uma perspectiva puramente mecânica:

Power creep significa que quando fazemos uma carta melhor do que as cartas antigas, ela é simplesmente mais poderosa, ou fazemos uma carta que aumenta o nível geral de poder do jogo e melhora também as cartas antigas. Por exemplo, quando lançamos Grim Patron, de repente o Warsaw Commander e Battle Rage, cartas que eram pouco utilizadas antes, (...) se tornaram mais poderosas. (HEARTHSTONE, 2015, Tradução do autor ${ }^{13}$ )

Ben Brode deixa de mencionar que o lançamento de cartas mais poderosas não só produz um efeito de aumento de poder no jogo, mas também mobiliza o surgimento de novos decks de alto desempenho e competitividade. Como fica claro no vídeo do Extra Credits, o lançamento de novas cartas força jogadores competitivos a comprar pacotes das novas expansões. Para Ben Brode é fundamental que cartas mais poderosas sejam

12 Citação original: There is some things that really prevents new players from playing Hearthstone and one of those things is the amount of money and the amount of commitment you really need to get to an acceptable level to play somewhat of a fair game of Hearthstone. This is something that Blizzard constantly overlooks with every single expansion. With much more cards to get, with the cards being hard to access in different types of card packs (...) With every single expansion every single player that plays Hearthstone will have a harder time catching up, a harder time meeting an adequate level of fair play. It really sucks for them.

13 Citação original: Power creep means when we either make a card that is better than old cards, it is just more powerful, or we make a card that kinda increases the overall power level of the game and make old cards better. For example, when we released Grim Patron, all of the sudden Warsaw Commander and Battle Rage, underplayed cards before (...) became more powerful. 
lançadas ao longo do tempo, senão o comprometimento da Blizzard, de manter o jogo sempre excitante, não ocorre.

Noxious posiciona seu desconforto com a postura da Blizzard, que trata o problema puramente enquanto de balanceamento no game design. Segundo sua linha de pensamento, em um jogo digital não haveria nenhuma dificuldade em alterar uma carta antiga, ao contrário de card games analógicos. O lançamento de $\mathrm{EH}$ (carta paga), por exemplo, torna BBB (carta gratuita) obsoleta indefinidamente. Certamente as decisões da Blizzard sobre o lançamento de conteúdo inédito não são tomadas somente por uma perspectiva de balanceamento. Como o próprio Noxious aponta, o fenômeno deriva de uma instrução intencional no design do jogo:

(...) Um aumento vertiginoso no nível de poder do conteúdo em comparação com conteúdo antigo. (...) À medida que o novo conteúdo é lançado, o conteúdo mais antigo fica obsoleto. Isso pode ser projetado ou evitado. Ou, às vezes, é um resultado não intencional do design de jogos, alguém tenta criar um conteúdo que deveria ser incrível, mas acaba tornando todo o resto obsoleto (...). (NOXIOUS, 2015, Tradução do autor $\left.{ }^{14}\right)$

As múltiplas vozes colocam em perspectiva diferentes camadas do power creep. É evidente que a Blizzard mobiliza um poderoso aparato midiático para a naturalização da serialização em sua lógica de expansões, ora narrativizando a problemática, ora tratando como questões de balanceamento. A flutuação do metagame, entretanto, nos ajuda a localizar o power creep em uma dimensão material, inscrita no game design do jogo, mas que politiza a filosofia da Blizzard e a própria ideia de serialização. A fala dos jogadores e comentadores, por sua vez, revela pistas que escapam do argumento hegemônico postulado pela Blizzard, em uma espécie de resistência às práticas mercadológicas da corporação. A desvelação dos agenciamentos e materialidades que

14 Citação original: (...) a creeping increase in power level in content related to older content. (...) As new content is released, older content becomes obsolete. This can be design for or against. Or sometimes it's an unintentional result of game design, somebody tries to make a piece of content that is supposed to be so awesome, but it renders everything else obsolete (...). 
produzem o power creep, portanto, é fundamental para a politização da prática.

\section{Considerações finais}

Pesquisas sobre relações entre consumo, bens virtuais, ambientes de jogo de alta competitividade (eSports) e capitalismo tardio já começam a surgir internacionalmente (GIDDINGS; HARVEY, 2018). Embora ainda escassas no Brasil, é possível localizar outros trabalhos que avançam na discussão. Entretanto, muitos ainda se ocupam em tratar o fenômeno por um viés do consumo simbólico (MACEDO; VIEIRA, 2017), destacando assim o papel que os bens virtuais ocupam na construção de sentidos a partir do acoplamento jogador-jogo, ou oferecendo uma taxonomia inicial para o tratamento de bens virtuais em jogos competitivos (MACEDO; VIEIRA, 2018). Entretanto, acreditamos que ainda não há uma preocupação geral em politizar as maquinações que, antes de mais nada, dão origem aos ditos bens virtuais. Nesse sentido, o mapeamento desenvolvido anteriormente (FALCÃO; MARQUES, 2017) foi fundamental para revelar os rastros que deram origem a este trabalho.

Acreditamos, portanto, que esta pesquisa apresenta algumas contribuições gerais para os game studies, bem como para os estudos em midiatização e consumo. Em primeiro lugar, buscamos politizar a indústria cultural do entretenimento na contemporaneidade, localizando a ação das estruturas agenciais do capitalismo tardio. O cenário competitivo e profissional de videogames está em sua infância, bem como as estratégias político-econômicas que subjazem esse mercado. Esse fato torna urgente a localização das estruturas agências e dispositivos materiais-discursivos (BARAD, 2007) que possibilitam a ação do capitalismo tardio. É certo que os fenômenos de midiatização e serialização ganham novos contornos no campo dos videogames, sendo o power creep uma de suas conformações. É preciso também iluminar e politizar outras práticas, como sistemas de microtransações, loot boxes, remuneração a partir de streaming etc. 
Por fim, cabe apontar que a presente pesquisa corrobora não só com a questão do consumo em videogames, mas parte de uma perspectiva bottom-up que produz questões sobre o estado ontológico e epistemológico do que se entende por jogo. A conformação dessa rede de consumo, apropriação e técnica em jogos coloca em causa (a) o que passa a ser percebido como jogo/nãojogo e (b) de que forma temos acesso a esses fenômenos. Desdobramentos e efeitos dessa rede são sentidos com cada vez mais intensidade, seja na demissão em massa de trabalhadores, em boicotes realizados por profissionais a campeonatos até movimentos sociais em prol da sindicalização na indústria. Essas pistas apontam para a urgência na politização e problematização da questão.

\section{Referências}

AARSETH, E. A Hollow World. World of Warcraft as Spatial Practice. In: H. G. CORneliussen, H. G.; RETTBERG, J. W. (Eds.). Digital Culture, Play, and Identity. A World of Warcraft Reader. Cambridge: The MIT Press, 2009.

BAILEY, Hearthstone player count has grown by 30 million in the past 18 months. PCGamesN, 2018. Disponível em: <https://www.pcgamesn.com/hearthstone/ hearthstone-player-count>.

BARAD, K. Meeting the Universe Halfway. Durham: Duke University Press, 2007.

BASTOS, M. T. Medium, media, mediação e midiatização: a perspectiva germânica. In: MATTOS, M.; JANOTTI, J.; JACKS, N. (Orgs.) Mediação ๒ Midiatização. Salvador: Edufba, 2012. p. 53-77.

BATESON, G. A Theory of Play and Fantasy. In: BATESON, G (Ed.). Steps to an Ecology of Mind. New York: Ballantine Books, 1972.

BRAGA, J. L. Interaction as a context of communication. Matrizes, São Paulo, v. 6, n. 1-2, p. 25, 11 dez. 2012.

COULDRY, N. Theorising media as practice. Social Semiotics, Londres, v. 14, n. 2, p. 115-132, ago. 2004.

COULDRY, N.; HEPP, A. The mediated construction of reality. Cambridge, UK: Polity Press, 2016.

FALCÃO, T. Uma incursão sobre as estruturas comunicacionais em mundos virtuais: estudo sobre a mediação dos diálogos pela figura do jogo. Dissertação de Mestrado - Programa de Pós-Graduação em Comunicação e Cultura Contemporâneas, UFBA. 2010. 
FALCÃO, T.; MARQUES, D. Pagando para vencer: Cultura, Agência e Bens Virtuais em Video Games. Contracampo, Niterói, v. 36, n. 2, p. 133-156, ago./out. 2017.

GIDDINGS, S.; HARVEY, A. Introduction to Special Issue Ludic Economies: Ludic Economics 101. Games and Culture, Ahead of Print, 11 fev. 2018.

HEARTHSTONE. Designer Insights with Ben Brode: The Dark Side of Releasing New Content. YouTube, 2015. (6m53s). Disponível em: <https://youtu.be/EGc6hAr8r6c>.

HEPP, A. The communicative figurations of mediatized worlds: Mediatization research in times of the 'mediation of everything'. European Journal of Communication, v. 28, n. 6, p. 615-629, dez. 2013.

HJARVARD, S. Mediatization: conceptualizing cultural and social change. Matrizes, São Paulo, v. 8, n. 1, p. 21, 24 jun. 2014.

KRIPPARRIAN. [Hearthstone] Ben Brode \& Kripp On Bad Cards. YouTube, 2015a. (30m46s). Disponível em: <https://youtu.be/flioYlKO79A>.

KRIPPARRIAN. [Hearthstone] How I'd Improve HS. YouTube, 2015b. (21m12s). Disponível em: <https://youtu.be/Cs3H5NYQKVo>.

KRIPPARRIAN. [Hearthstone] Power Creep \& Buffing Bad Cards. YouTube, 2015c. (14m22s). Disponível em: <https://youtu.be/Ov4P8rSIPjw >.

JAMESON, F. Postmodernism, or, The Cultural Logic of Late Capitalism. London: Verso, 1991.

JUUL, J. Half-Real. Cambridge, Massachusetts: MIT Press, 2005.

LATOUR, B. Reagregando o social. Salvador: EDUFBA, 2012.

REUTERS. Hearthstone announces $\$ 4$ million prize pool in 2019. ESPN, 2018. Disponível em: < https://www.espn.com/esports/story/_/id/25410787/ hearthstone-announces-4-million-prize-pool-2019>.

MACEDO, T.; VIEIRA, M. D. C. Dinâmicas de consumo de bens virtuais: práticas e valores no universo de League of Legends. E-Compós, v. 21, n. 1, 26 abr. 2018.

. Muito além dos pixels: experiências de consumo e cultura material em League of Legends. Comunicação Mídia e Consumo, São Paulo, v. 14, n. 41, p. 146, 15 dez. 2017.

NOXIOUS. POWER CREEPIN' MY HEARTHSTONE. YouTube, 2015. (17m30s). Disponível em: https://www.dropbox.com/s/51lltclklqysawy/powercreep8.mp4?dl=0.

NOXIOUS. Thoughts on Power Creep in Hearthstone. YouTube, 2014. (26m08s). Disponível em: <https://www.dropbox.com/s/h0xs8xldogt536k/powercreep6. mp4?dl=0>.

TURTIAINEN, R.; FRIMAN, U.; RUOTSALAINEN, M. "Not Only for a Celebration of Competitive Overwatch but Also for National Pride": Sportificating the Overwatch World Cup 2016. Games and Culture, Ahead of Print, 27 ago. 2018.

VERÓN, E. Mediatization theory: a semio-anthropological perspective and some of its consequences. Matrizes, São Paulo, v. 8, n. 1, p. 13, 24 jun. 2014. 


\section{Sobre os autores}

Thiago Falcão - Professor Adjunto do Curso de Comunicação em Mídias Digitais e Coordenador do Programa de Pós-Graduação em Comunicação da Universidade Federal da Paraíba. É doutor em Comunicação e Cultura Contemporâneas pela Universidade Federal da Bahia e tem Pós-Doutorado em Comunicação e Audiovisual pela Universidade Anhembi Morumbi. Sua pesquisa se dedica a compreender as formas agenciais do capitalismo tardio no entretenimento, problematizando o papel do jogo na compreensão da sociedade contemporânea . No presente artigo, atuou na concepção e desenho geral da pesquisa, desenvolvimento e revisão da discussão teórica, redação e revisão do manuscrito final.

Daniel Marques - Professor assistente do Centro de Cultura, Linguagem e Tecnologias Aplicadas da Universidade Federal do Recôncavo da Bahia (CECULT/ UFRB), doutorando em Comunicação e Cultura Contemporâneas (Póscom/ UFBA), pesquisador no Lab404/UFBA e Caminhos da Criação/UFRB, coordenador do LAG - Laboratório de Análise e Desenvolvimento de Games (CECULT/UFRB). Mestre em Cultura e Sociedade pelo Programa Multidisciplinar de Pós-Graduação em Cultura e Sociedade (PósCultura/UFBA). Especialista em Design Estratégico (UNIFACS/LAUREATE) e bacharel em Design (UNIFACS/LAUREATE). No presente artigo, atuou no desenvolvimento da metodologia, coleta e interpretação dos dados, desenvolvimento e revisão da discussão teórica, redação e revisão do manuscrito final.

Data de submissão: 10/10/2018

Data de aceite: 04/10/2019 